\title{
Archéopages Archéopages
}

Archéologie et société

\section{L'exécution de traîtres à la cause révolutionnaire en 1794. Découvertes archéologiques au Morne à Savon en Guadeloupe}

Thomas Romon, Christian Stouvenot et Gérard Lafleur

\author{
CpenEdition \\ Journals \\ Édition électronique \\ URL : https://journals.openedition.org/archeopages/542 \\ DOI : 10.4000/archeopages.542 \\ ISSN : 2269-9872 \\ Éditeur \\ INRAP - Institut national de recherches archéologiques préventives \\ Édition imprimée \\ Date de publication : 1 septembre 2014 \\ Pagination : 40-45 \\ ISSN : 1622-8545 \\ Référence électronique \\ Thomas Romon, Christian Stouvenot et Gérard Lafleur, "L'exécution de traîtres à la cause \\ révolutionnaire en 1794. Découvertes archéologiques au Morne à Savon en Guadeloupe », \\ Archéopages [En ligne], 39 | 10/2013-01/2014, mis en ligne le 01 janvier 2016, consulté le 22 janvier \\ 2022. URL : http://journals.openedition.org/archeopages/542 ; DOI : https://doi.org/10.4000/ \\ archeopages. 542
}




\section{L'exécution de traîtres à la cause révolutionnaire en 1794 \\ Découvertes archéologiques au Morne à Savon en Guadeloupe}

Thomas Romon Inrap, LMR S199-PACEA

Christian Stouvenot Ministère de la Culture et de la Communication, UMR 8096-ArchAm «Archéologie des Amériques »

Gérard Lafleur Socitete dhistorie de la Guadeloupe

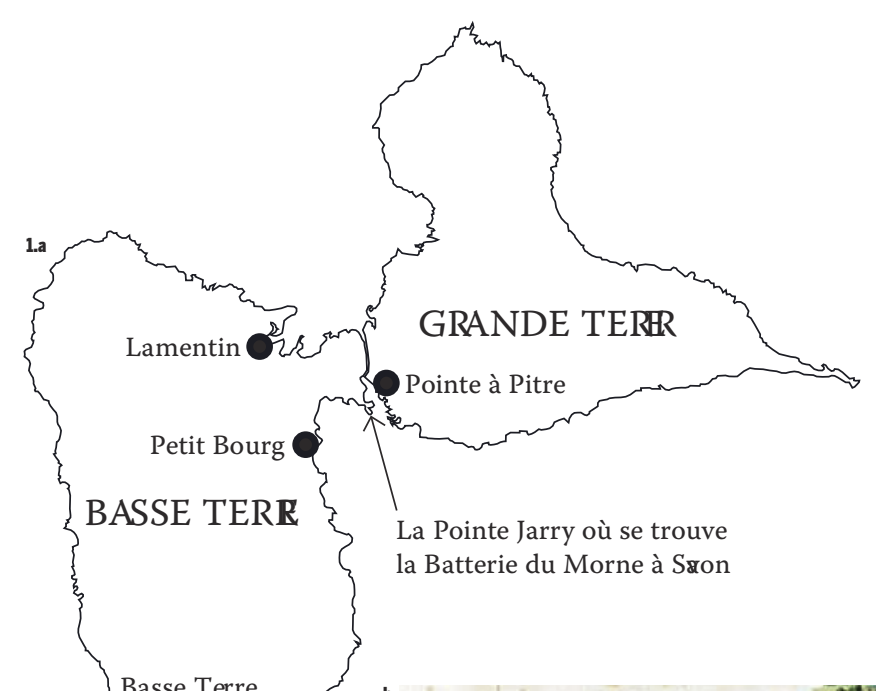

1a. Le Morne à Savon est situé sur la Pointe Jarry, en face de la ville de

Pointe-à-Pitre-Carte des îles de Basse-Terre et Grande-Terre de Guadeloupe indiquant les localités citées (fond IGN). 1h. Vue de la rade de Pointe-à-Pitre vers 1773 1. Batterie du Morne à Savon ;

2 Pointe-à-Pitre : 3. Fort Louis ;

4. Îlet à Cochons;

5. Pointe à Martin ;

6. Pointe de la Gabarre;

7. Habitation Berville

où les Anglais établirent leur camp. Plan de Talsy.

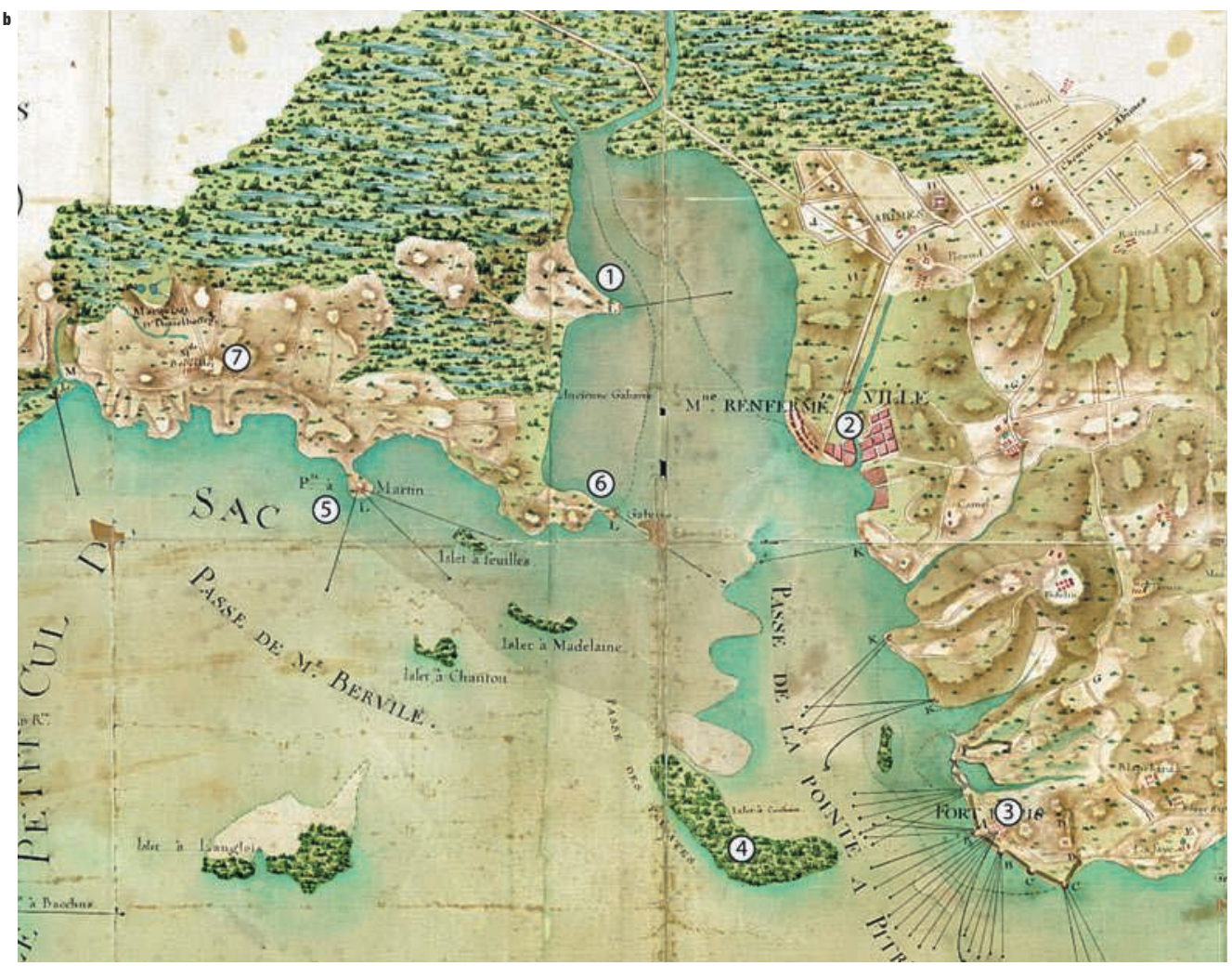


L'an II de la République, soit l'année 1794 du calendrier grégorien, est en Guadeloupe le théâtre d'événements très meurtriers, pas seulement du fait des combats entre les forces anglaises (alliées aux planteurs royalistes) et les troupes républicaines, mais aussi à cause des représailles sanglantes que les républicains infligèrent aux royalistes, tant pour les punir de leur alliance opportuniste avec les Anglais, que pour l'exemple par la terreur. Les planteurs royalistes payèrent très cher d'avoir négocié l'appui de l'Angleterre pour conserver leurs prérogatives sociales et économiques outre-mer. Plusieurs centaines d'entre eux furent exécutés à la batterie du Morne à Savon le 16 vendémiaire de l'an II, « 7 octobre 1794 vieux style».

En 1999, des prospections ont permis de vérifier la présence d'ossements humains dans la pente est de ce morne ${ }^{1}$ (Stouvenot, 1999). L'hypothèse est alors faite qu'il pourrait s'agir des vestiges de l'exécution de 1794. Une petite opération de sondages a donc été réalisée en avril 2004 (Romon, Stouvenot, 2004). Le Morne à Savon est une petite élévation en bordure ouest de la rade de Pointe-à-Pitre [ill. 1]. Situé sur la commune de Baie-Mahault, à l'est de la Pointe Jarry, il se présente aujourd'hui comme un petit plateau de forme triangulaire qui domine la rade à $6 \mathrm{~m}$ d'altitude. Si plusieurs témoignages oraux indiquent que la présence de squelettes dans ce secteur était connue de longue date, aucune trace tangible ne permettait d'en préciser les caractéristiques. Il n'était notamment pas établi que ces vestiges aient un lien avec les événements de 1794, d'autant que le site est implanté sur une ancienne batterie, lieu de combat par excellence, mais aussi à proximité d'un ancien cimetière figuré sur les cartes IGN au 1/20 ooo 2 .

\section{Les faits historiques}

Après la restitution de la Guadeloupe à la France au traité de Paris qui mit fin à la guerre de Sept Ans3, les autorités françaises ont revu en totalité le système de défense de leurs colonies ${ }^{3}$. Pointe-à-Pitre ayant pris de l'importance, on décida de fortifier son accès et ses abords. La batterie du Morne à Savon est située sur un petit morne qui borde la Rivière Salée, de l'autre côté de la rade de Pointe-à-Pitre, face à la ville [ill. 1]. Elle fut créée sur l'ordre du gouverneur De Nolivos lors de sa tournée de 1768 (Archives départementales de la Guadeloupe, $1 \mathrm{Mi}$ 363, 23/6/1768. Objet des batteries de la Basse-Terre et Grande-Terre Guadeloupe avec leurs plans et le figuratif des côtes sur lesquelles les anciennes et celles nouvellement construites se trouvent posées..., Bibliothèque Mazarine, Ms 3451). En forme de demi-lune, elle fut armée de deux pièces d'artillerie qui permettaient de croiser ses feux avec ceux des batteries de lî̂let à Cochons, situés au sud de la rade, d'une part, et avec ceux de la batterie du Morne Renfermé, installés dans la ville,

d'autre part.
Mais c'est surtout aux premiers temps de la République que la batterie du Morne à Savon joua un rôle majeur. Après que la Martinique leur fut livrée par les colons, les Anglais débarquèrent à Gosier en avril 1794. Avec l'aide des royalistes, ils prirent la Guadeloupe. En juin, une armée patriote d'un millier d'hommes fut envoyée de France pour reprendre les possessions françaises avec à son bord Victor Hugues, commissaire de la Convention. Elle débarqua en Grande-Terre avec le décret d'abolition de l'esclavage du 16 pluviôse an II (Décret $\mathrm{n}^{\circ} 2262$ de la convention nationale : « La Convention Nationale déclare que l'esclavage des Nègres dans toutes les colonies est aboli [...] », proclamé le 7 juin 1794 en Guadeloupe [Archives nationales d'outre-mer, $\mathrm{C}_{7} \mathrm{~A} 47$, fol. 8]) qui permit de recruter plus de 3000 "Noirs » et de chasser Anglais et royalistes de l'autre côté de la Rivière Salée. De fait, la Guadeloupe est alors divisée, Français républicains en Grande-Terre et Anglais et royalistes en Basse-Terre. Ceux-ci aménagèrent un camp retranché, le camp Berville, sur la Pointe Jarry. Ils armèrent fortement les batteries qui faisaient face à Pointe-à-Pitre dans laquelle se trouvaient les troupes révolutionnaires. Les deux batteries de Morne à Savon et de Saint-Jean, appuyées par trois chaloupes canonnières, tirèrent beaucoup de «boulets rouges » sur la ville afin de provoquer des incendies et ceci pendant 30 jours (Archives nationales d'outre-mer, Dépôt des fortifications des colonies, Guadeloupe, dossier n ${ }^{\circ} 450$, Extrait d'une lettre des commissaires délégués par la Convention nationale aux Îles du vent, 4 Thermidor an 2 [25 juillet 1794] ; Lacour, 1857, t. II, p. 314-515).

Le 28 septembre, en effectuant une manœuvre d'encerclement par le Lamentin et Petit Bourg, les troupes révolutionnaires isolèrent les Anglais au camp Berville. Totalement coupé du reste de la colonie, le général anglais, afin de sauver ses troupes, négocia la possibilité d'embarquer sur les navires anglais pour quitter la Guadeloupe. En contrepartie, il livra les troupes françaises qui combattaient à ses côtés : « Les Anglais sont faits prisonniers après avoir eu les honneurs de la guerre, conformément à l'article $1^{\text {er }} \mathrm{de}$ la capitulation. Tous les Français qui sont pris avec eux subissent la peine prononcée contre les traîtres à la patrie... » (Archives nationales d'outre-mer, Dépôt des fortifications des colonies, dossier 451). Ceux-ci furent immédiatement conduits à la batterie du Morne à Savon et fusillés sur place. L'historien Auguste Lacour (Lacour, 1857), souvent bien informé sur les événements de cette époque, en fait le récit suivant : « Les émigrés destinés à la mort n'avaient pas besoin d'être jugés, il suffisait de constater qu'ils avaient été pris les armes à la main. Chavidan, chef de bataillon, présida la commission à qui fut donnée cette douloureuse mission. Cette formalité accomplie, les émigrés furent conduits au morne Savon, placés sur le bord des fossés des batteries et fusillés. En recevant la mort ils tombaient dans les fossés. On n'avait pas la peine
1. En créole guadeloupé
le terme morne désigne

2. Ces cartes de la série donne Saint-Vincent, la Dominique, Grenade et Sainte Lucie, la Martinique,

la Guadeloupe et SaintDomingue aux Français. 


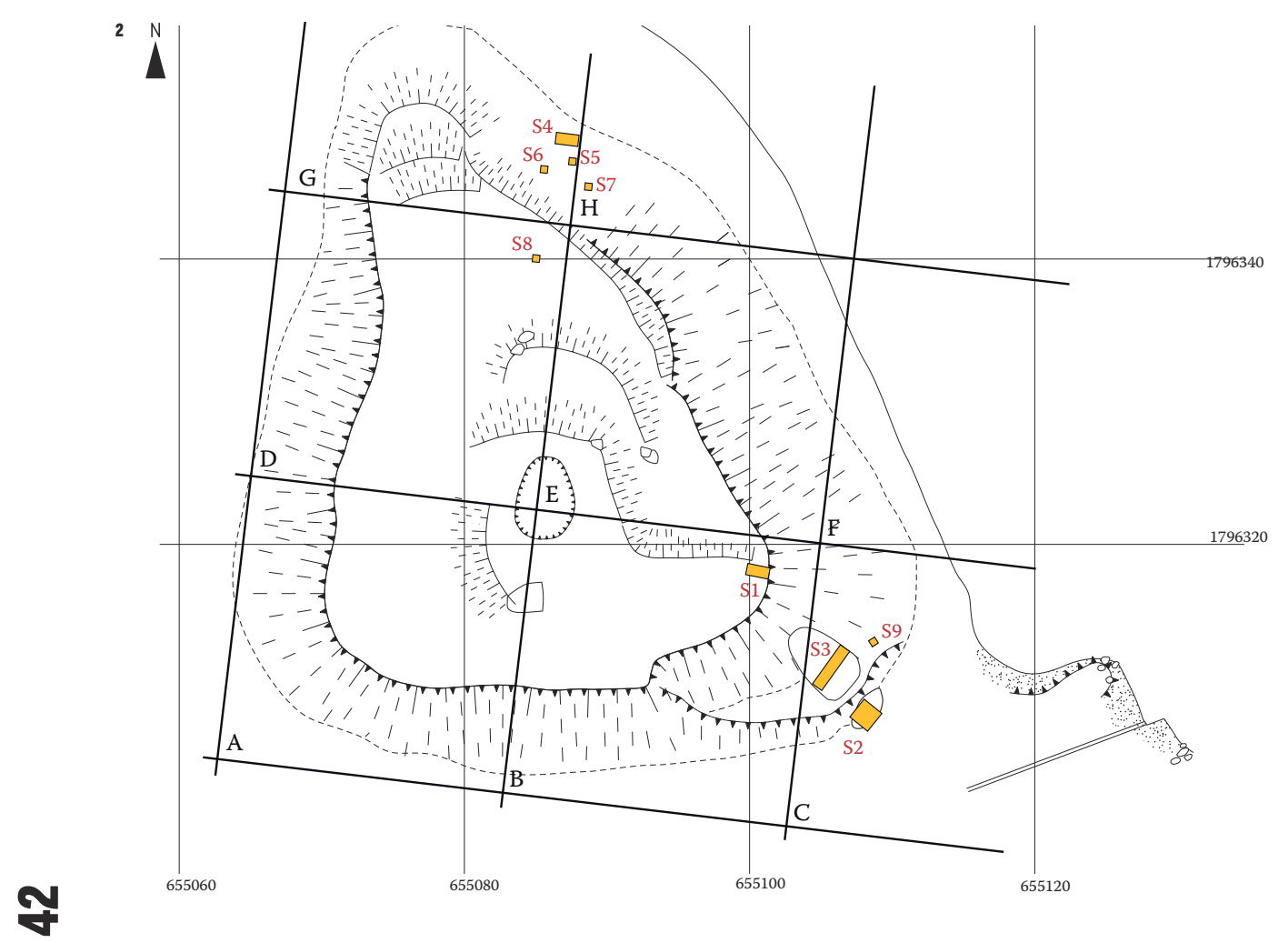

2. Relevé topographique du Morne à Savon réalisé au cours de l'opération de 2004. Le sondage 4 qui a livré des vestiges humains est situé dans le secteur nord-est (carre G). Les autres sondages sont négatifs (carroyage local pour le terrain et recalage sur I'UTM20)
3. Le sondage 4, relevé et photographies

des vestiges humains.

Quatre individus sont

représentés par des

portions de leur squelette

en connexion (en gris :

les portions d'individu

en connexion ; en blanc

les os en position

secondaire; en pointillé :

le front d'érosion).

Ind 3
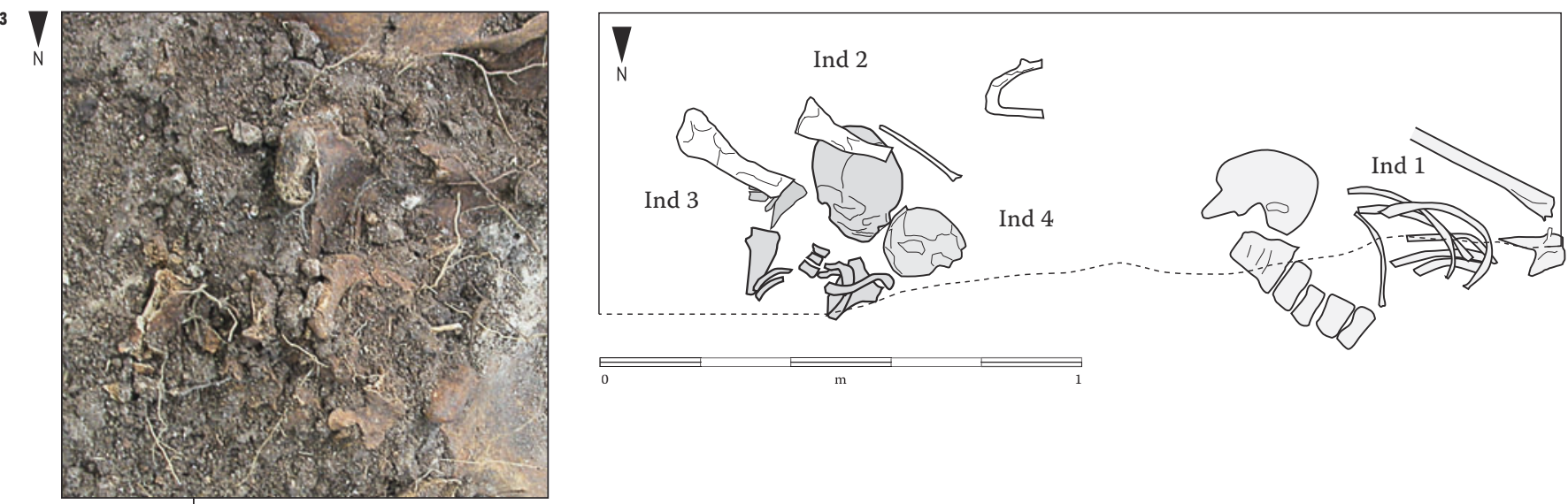

Е

m

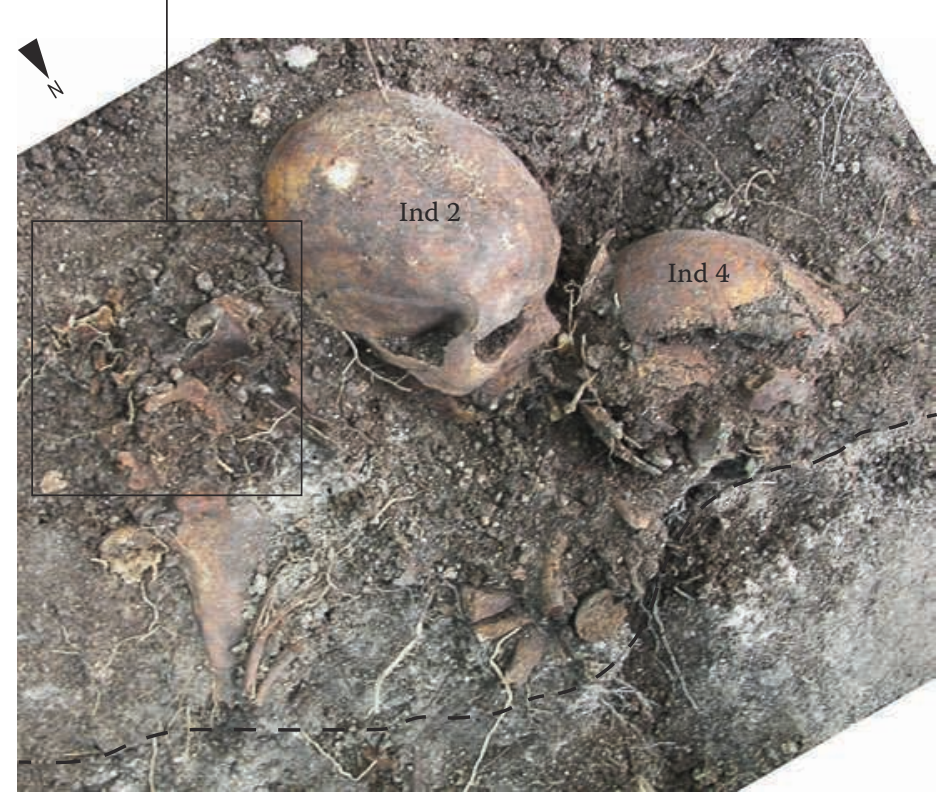

Ind 1

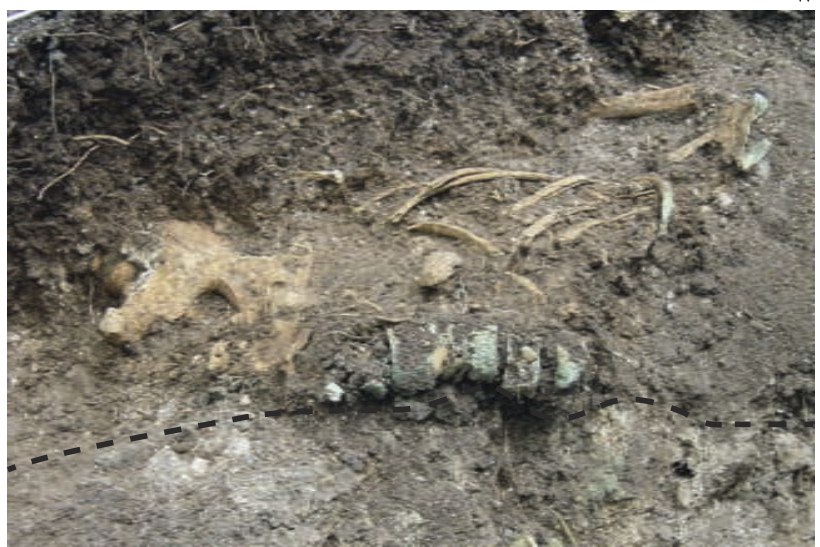


de les y descendre. Plusieurs n'étaient que blessés ; la terre jetée sur les victimes acheva ce que le plomb avait commencé » (Lacour, 1857, t. II, p. 337).

Henry de Poyen-Bellisle dans son ouvrage La guerre des Antilles, paru à la fin du XIX ${ }^{\mathrm{e}}$ siècle, donne d'autres précisions. Après la capitulation acceptée par le général anglais, « 14 oo Anglais se rendent avec les honneurs de la guerre et sont renvoyés comme prisonniers sur parole sur les navires de l'escadre. Les émigrés français livrés sont au nombre de 600 environ dont 300 blancs, 100 libres de couleur et 200 nègres esclaves. Les 400 premiers furent aussitôt fusillés » (PoyenBellisle, 1896, t. 1, p. 83). Cela est également confirmé par le courrier administratif. Un bilan établi après les événements recense 1309 soldats et 80 officiers anglais prisonniers et, dans la rubrique «Contrerévolutionnaires », il distingue «Contrerévolutionnaires armés servant dans l'armée anglaise livrés par le général Colin Graham, commandant du camp Berville : Guillotinés : 140 ; Fusillés : 363 ; Condamnés à la chaîne dont 36 femmes, tous des Noirs, pour avoir servi l'armée anglaise dans le camp Berville : 362 » Archives nationales d'outre-mer, $\mathrm{C}_{7} \mathrm{~A}$ 47, fol. 94 ; Archives départementales de la Guadeloupe, 1 Mi 106, État des munitions..., Non daté).

À la suite de la capitulation anglaise, la Guadeloupe fut totalement reprise par les révolutionnaires début décembre. Ceux-ci menèrent alors des actions en direction des colonies françaises des Petites-Antilles prises par les Britanniques. Les armées révolutionnaires vont temporairement occuper Sainte-Lucie, Grenade, Saint-Vincent, Saint-Martin et Sainte-Croix. Cependant, hormis pour la République d'Haïti, devenue indépendante en 1804, l'esclavage est rétabli dans l'ensemble de la Caraïbe dès le 17 juillet ${ }_{1802}{ }^{4}$ et la Guadeloupe fut reprise par les Anglais le 10 août $1810^{5}$.

\section{L'analyse des ossements et du mobilier associé}

Lensemble du morne est couvert d'une végétation arborée assez jeune. Ses versants sont abrupts et présentent une pente souvent supérieure à $50^{\circ}$. Ils sont faiblement entamés par l'érosion naturelle et les colluvions qui en sont issues nappent leur base. Les côtés ouest et sud sont entaillés par des aménagements récents : canal, chemin et canalisations enterrées. Une végétation de mangrove frange la bordure avec la rade sur le côté est. Une inspection du morne a permis de positionner tous les restes humains visibles en surface et d'implanter quelques sondages de reconnaissance [ill. 2] sur les secteurs paraissant les plus prometteurs en termes de potentiel anthropologique.

Le côté nord-est, moins anthropisé, plonge vers la rade de Pointe-à-Pitre où il est bordé de mangrove. C'est sur ce versant que les ossements humains apparaissent. Des sondages manuels ont été entrepris à cet endroit. Un seul a livré des vestiges en connexions. Il s'agit du sondage 4, qui mesure $2 \mathrm{~m}$ par $60 \mathrm{~cm}$. Il est localisé en bas de pente, juste en amont du front d'érosion. Son grand axe est perpendiculaire à la pente (est-ouest). Les autres sondages de ce secteur, au nombre de huit et qui totalisent $5 \mathrm{~m}^{2}$, n'ont pas livré de restes humains. Ils permettent de circonscrire l'extension maximale de la zone susceptible de contenir ces vestiges.

Une partie des ossements humains sont en position secondaire, dispersés sur toute la surface du sondage. L'autre partie, sous la première, se présente en concentrations et montre des connexions anatomiques. La première concentration est localisée à l'extrémité occidentale du sondage. Elle a livré les restes d'une portion d'individu en connexion (l'individu 1). La seconde est située à l'extrémité orientale du sondage. Ce sont les vestiges de deux portions d'individus en connexion (les individus 2 et 3 ) et peut-être d'un troisième (l'individu 4) représenté par son calvarium ${ }^{6}$ [ill. 3].

Les ossements en position secondaire sont situés dans la partie supérieure du sondage, épars, au-dessus des portions d'individus en position primaire. Ce sont de petits éléments (phalanges, patella) mais aussi des éléments plus grands (un fémur, un humérus, un tibia, une mandibule). Ils sont issus de migrations dans la pente et proviennent probablement de plus haut (colluvionnement). Ils appartiennent à un épisode chrono-stratigraphique différent et postérieur à celui des portions d'individus en position primaire.

L'individu 1 est un adulte de sexe masculin. Il repose sur le dos, plus ou moins à l'horizontale, perpendiculairement à la pente, tête à l'ouest. Il est représenté par une partie de sa scapula droite, son humérus droit, six côtes droites, ses vertèbres lombaires de la deuxième à la cinquième, très mal conservées, son sacrum également très mal conservé, son coxal droit et son fémur droit. L'humérus et le fémur sont pour partie dans la coupe sud du sondage. Le côté gauche n'est pas conservé, il a été détruit par l'érosion de pente. Aucune limite de creusement de fosse n'a été détectée à la fouille. La mise à plat de certaines parties osseuses (scapula, côtes et bassin), ainsi que la préservation de connexions anatomiques laissent penser que la décomposition s'est effectuée sans trop de contrainte, probablement du fait d'un enfouissement très superficiel du cadavre. La présence de petits éléments en position secondaire est probablement liée à la migration dans la pente de ces petits éléments. Ceux-ci n'appartiennent pas forcément à ce même individu.

L'individu 2 est un adulte de sexe indéterminé. Il repose sur le dos, dans le sens de la pente et avec un pendage à peu près identique (environ $45^{\circ}$ ), tête au sud (le haut de la pente). Il est représenté par son crâne, son rachis jusqu'à la troisième vertèbre thoracique, des fragments des côtes supérieures droites, les trois premières côtes gauches, ses scapulas ainsi que les extrémités proximales de ses humérus droit et gauche et sa clavicule gauche. Le reste du cadavre n'est pas conservé, détruit par 


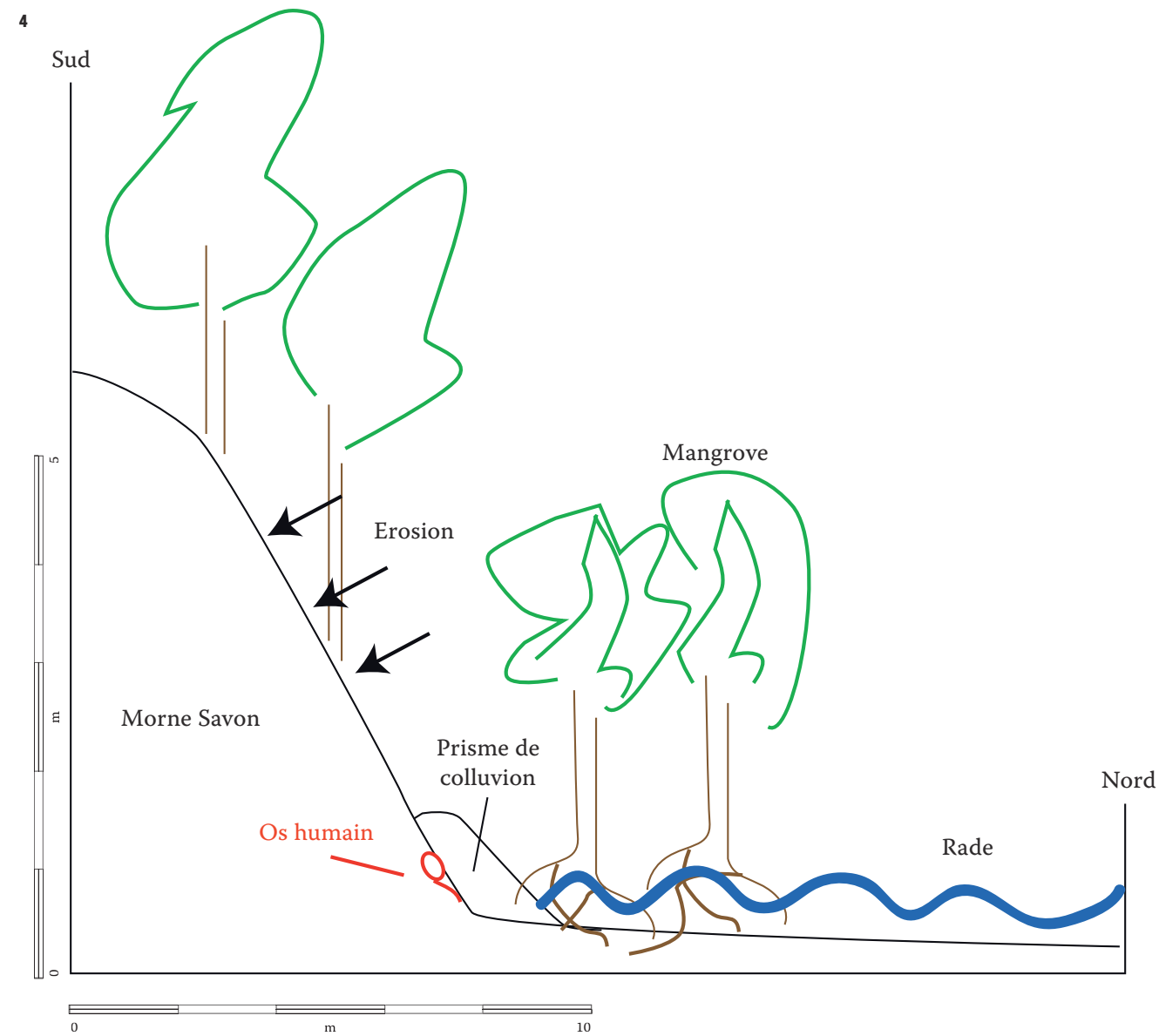

l'érosion de pente. Aucune limite de creusement de fosse n'a été détectée à la fouille. Malgré de légères migrations en blocs dans la pente, la mise à plat des scapulas et des côtes et la position cohérente des différents éléments anatomiques amènent à conclure, comme pour l'individu précédent, à un enfouissement assez superficiel du cadavre.

L'individu 3 est un adulte de sexe indéterminé. Il repose pour partie sur l'individu 2, sur le dos, dans le sens de la pente, tête au sud (le haut de la pente), selon un pendage à peu près identique à celui de la pente (environ $45^{\circ}$ ) et identique à celui de l'individu 2. Il est représenté par son rachis, de la première à la quatrième vertèbre thoracique, très dégradé, par sa scapula gauche et sa deuxième côte gauche. Le reste de cet individu n'est pas conservé, détruit par l'érosion de pente. Aucune limite de creusement de fosse n'a été détectée à la fouille. Cet individu est très partiellement conservé. Ses restes conservés sont localisés dans l'espace situé entre l'épaule et le crâne de l'individu 2. Il est possible que ce soit l'individu 3 qui soit à l'origine de la rotation du crâne de l'individu 2, ce qui indiquerait la simultanéité de ces deux inhumations.

L'individu 4 est un adulte de sexe indéterminé. Il est uniquement représenté par sa voûte crânienne reposant sur sa face latérale gauche, à l'ouest du crâne de l'individu 2. Tout le reste de cet individu est détruit par l'érosion de pente. La position primaire ou secondaire de cet élément est discutable.
Dans la première hypothèse, l'individu repose directement à la gauche de l'individu 2, et la simultanéité des inhumations des individus 2 et 4. peut être proposée.

Le sondage 4 a livré trois monnaies amalgamées dans un contenant en bois conservé par les oxydes de cuivre. Ce matériel, associé à l'individu 2, est en cours d'étude. Les monnaies ont été identifiées ${ }^{7}$, ce sont trois « deux sous de Cayenne », frappés en 1789. Ces monnaies en alliage argent-cuivre sont produites sous Louis XVI à Cayenne, mais pour le compte de toutes les colonies d'Amérique. Elles permettent de rattacher les restes humains découverts à la fin du XVIII ${ }^{\mathrm{e}}$ siècle.

\section{Conservation et nature du dépôt funéraire}

Cette opération a permis de mieux comprendre le processus de dégradation du Morne à Savon. Les pentes de ses flancs sud et ouest sont très altérées par l'activité anthropique, et ce dès les années 1960 avec la mise en place de la zone industrielle de Jarry-Houelbourg. Seules les pentes est et nord-est présentent encore des dépôts non affectés par les travaux et susceptibles de contenir des vestiges datant du massacre de 1794. Ce secteur est soumis à une érosion et à un colluvionnement tendant à une stabilisation de la pente (environ $45^{\circ}$ ). Ce phénomène est à l'origine du recul du sommet du talus : tous les vestiges osseux qui s'y trouvaient ont migré à la faveur de la pente très raide et 
se sont dispersés dans les parties basses. Dans le bas de la pente, on constate la mise en place d'un prisme de colluvions ${ }^{\mathbf{8}}$ recouvrant des vestiges humains en connexion et les préservant momentanément de l'érosion [ill. 4]. Malgré la présence d'une mangrove en bord de rade protégeant quelque peu les terrains, le pied de pente est soumis à la houle marine, ce qui provoque un recul érosif de la couverture colluviale, phénomène qui conduit à faire affleurer les vestiges humains enfouis.

La mise en place des sondages complémentaires autour du sondage 4 a permis de circonscrire une zone où la couche contenant les ossements humains est préservée. Cette zone correspond à une surface d'au moins $4 \mathrm{~m}^{2}$ située directement à l'est du sondage 4 . Il est cependant possible et vraisemblable que cette couche contenant les squelettes soit également conservée en d'autres endroits, en particulier vers la pointe nord et sur le flanc nord-est du morne. La vérification de cette hypothèse demanderait la mise en place d'une opération nettement plus importante que l'intervention de 2004.

L'érosion, qui a permis le repérage des vestiges humains, est également responsable de leur destruction. Les quatre individus identifiés, uniquement représentés par des portions de leur squelette, montrent une décomposition en espace colmaté différé (Duday, 1990), avec des mouvements amplifiés par la pente et la gravité. Il s'agit principalement de la mise à plat des ceintures scapulaires et pelviennes ainsi que du gril costal. Tous les déplacements observés sur les portions d'individus en position primaire sont dans les volumes corporels. Aucune limite de fosse de creusement n'a pu être décelée à la fouille. De plus, les corps découverts présentent le même pendage que la pente naturelle actuelle du morne. Enfin, pour trois individus, l'hypothèse d'un ensevelissement simultané peut être proposée.

Les observations réalisées, bien que se rapportant à des portions anatomiques restreintes, permettent de suggérer quelques interprétations. Ces sépultures ne peuvent pas provenir du cimetière de l'hôpital militaire de Pointe-à-Pitre. Celui-ci, utilisé de la fin du XVIII ${ }^{\mathrm{e}}$ siècle et durant le XIX ${ }^{\mathrm{e}}$ siècle, est situé à proximité du Morne à Savon comme l'indique Le commercial du 23 mars $1864^{\circ}$. Ce type de cimetière est assez bien connu en Guadeloupe (Kacki, Romon, 2013) et présente une organisation qui ne correspond absolument pas à ce qui a été découvert ici ${ }^{\mathbf{1 0}}$. Au contraire, les données acquises sont tout à fait compatibles avec l'hypothèse d'exécutions «sur le bord des fossés des batteries » en 1794. La faiblesse du nombre d'individus mis au jour n'est pas seulement imputable à la faible surface explorée, mais sans doute également à de possibles dispersions juste après le dépôt et à l'érosion des terrains. Il est aussi envisageable que certains corps aient été prélevés juste après

les exécutions pour être inhumés en d'autres lieux.
Les individus découverts semblent avoir été sommairement arrangés, directement contre la pente du morne, certains disposés dans le sens de la pente (individus 2, 3 et 4), la tête vers le sommet du morne, d'autres, comme l'individu 1 , orientés perpendiculairement à cette pente. Ils ont ensuite été recouverts de sédiment avant leur décomposition avancée.

Dans le contexte de la rivalité franco-britannique, les Anglais ont tenté de se rendre maîtres de possessions françaises des Antilles. Profitant des troubles révolutionnaires, ils initient une alliance d'intérêts avec les planteurs royalistes qui espéraient ainsi mettre à distance les républicains, opposants aux principes esclavagistes. En Guadeloupe, le renforcement des troupes patriotes par l'intégration des « Noirs » a permis de conserver ce territoire et de promulguer l'abolition de l'esclavage voté quelques mois plus tôt par la Convention.

Dans ce cas particulier, l'archéologie se présente comme un complément à des données textuelles plutôt bien établies. Ce complément ne concerne pas la teneur des événements eux-mêmes, mais plus la contextualisation du cadre topographique dans lequel s'est déroulée cette exécution. Cette opération archéologique, très limitée, permet d'appréhender les objectifs plausibles d'une exploration plus conséquente qui reste à mener : le statut, l'état sanitaire et l'appartenance ethnique des exécutés, la chronologie des exécutions, les armes utilisées, les gestes qui ont suivi le massacre, comme ceux se rapportant à la gestion des cadavres. Elle permet enfin d'illustrer le sort réservé aux planteurs royalistes et à leurs gens, vaincus par les troupes patriotes, et abandonnés par leurs alliés britanniques lors de l'invasion avortée de la Guadeloupe par les Anglais en 1794.
8. Ou prisme colluvial,

9. Nous remercions He

d'outre-mer, Gua 115/798. agricole, édition du «Article $1^{\text {er }}$ : aucun désormais dans l'intérieur de l'hôpital de Pointe-àni Morne à Savon, en ce qui concerne les militaires et tous autres décédés audit hôpital. Les inhumation auront lieu dans le cimetière communal. ». Il s'agit très probablement de «l'ancien cimetière indiqué sur la carte IGN au $1 / 20$ ooo de 1955 , à l'ouest du morne. 1o. Il s'agit presque exclusivement de sépultures individuelles, en pleine terre et en cercueil.
Références bibliographiques

DUdAY H., 1990, «Observations ostéologiques et décomposition du cadavre : sépulture colmatée ou en espace vide », Revue archéologique du Centre de la France, t. 29, fasc. 2, p. 193-196.

LACOUR M. A., 1857, Histoire de la Guadeloupe, t. 2 Basse-Terre, Impr. du gouvernement, $496 \mathrm{p}$.

KACKI S., ROMON T., 2013, « From burials to population identity: Archaeological appraisal of the status of a Lesser Antilles colonial cemetery (Baillif, Guadeloupe) », Antiquity, 87 (337), p. 829-839.

Poyen-Bellisle H. de, 1896, La guerre des Antilles de 1793 à 1815, Paris, Berger-Levrault, 452 p.

Romon T., STOUVEnOT C., 2004 , « Baie-Mahault, Morne à Savon ", Bilan scientifique régional, DRAC Guadeloupe, p. 18.

StouvenOt C., 1999, Inventaire des Sites archéologiques SIVOM Nord Basse-Terre Deshaies, Sainte-Rose, Lamentin, Baie-Mahault, Petit-Bourg, rapport final d'opération. n SRA 99, Basse-Terre, SRA Guadeloupe, $370 \mathrm{p}$. 